\title{
Double Hopf Bifurcation of a Hematopoietic System with State Feedback Control
}

\author{
Suqi $\mathrm{Ma}^{1}$ \\ ${ }^{1}$ College of Science, China Agricultural University, China \\ Correspondence: Suqi Ma, College of Science, China Agricultural University, Beijing 100083, China
}

Received: March 13, 2018 Accepted: March 28, 2018 Online Published: June 28, 2018

doi:10.5539/jmr.v10n4p116 URL: https://doi.org/10.5539/jmr.v10n4p116

\begin{abstract}
The dynamics of a system composed of hematopoietic stem cells and its relationship with neutrophils is ubiquitous due to periodic oscillating behavior induce cyclical neutropenia. Underlying the methodology of state feedback control with two time delays, double Hopf bifurcation occurs as varying time delay to reach its threshold value. By applying center manifold theory, the analytical analysis of system exposed the different dynamical feature in the classified regimes near double Hopf point. The novel dynamics as periodical solution and quasi-periodical attractor coexistence phenomena are explored and verified by numerical simulation.
\end{abstract}

Keywords: Double Hopf bifurcation, cyclical neutropenia, time delay

\section{Introduction}

Mathematically and Biologically, the discussion of the complex dynamics of hematopoietic cell model is ubiquitous and interesting due to its inherent highly nonlinear attribute. The hematopoietic stem cells(HSCs) originates from the bone marrow to proliferate and differentiate into all other types of blood cells. Underlying biomedical discpline, people introduced population dynamics to describe the relationship between cells compartments and the application of delay differential equations is the necessary useful tool on hand.

The definition of a HSC has two properties: self-renewal and multipotent differentiation to cells with different function capabilities. People set forth all kinds of mathematcical models with the good understanding of how introduce compartment cells of proliferating phase into comparment cells of nonproliferating phase. Therefore, HSCs system is build on the basis that both nonproliferating phase and proliferating phase can produce, by successive divisions, all types of blood cells(white cells, red blood cells and platelets). Mackeys model proposed at the end of the seventies describe a decoupled quiescent phase of HSCS to study the dynamics of HSCs and its related diseases (Mackey, 1978). Since then it has been improved and analyzed by many authors, including Mackey and coauthors (Pujo-Menjouet \& Mackey, 2004; Pujo-Menjouet et al., 2005; Fowler \& Mackey, 2002). In their models, delay is an salient factor in introducing HSCs population models. Proliferating cells can die with apoptosis and divide after a given time to produce two daughter cells, then immedieatelly enter a new nonproliferating phase.

Due to the dysfunction in regulatory control process of blood cell production, some haematological disease emerge and cyclical neutropenia $(\mathrm{CN})$ is the most extensively studied with low level oscillating phenomena happening. Medical observation have exhibited that the neutrophils fall down into abnormally level in a period about 19 to 21 days, and longer period happen in some patients with 40 to 80 days in human being. With the exception of period oscillating from 11 to 15 days, the similar oscillation character of cyclical neutropenia have been observed in the contrast experiment result of grey collie. With the assumption that neutrophils experience maturation time to release into circulation through the body, people have put forth the cyclical neutropenia dynamical system which is based on the negative feedack regulation mechanism (Fowler \& Mackey, 2002; Bernard et al., 2003; Santillan et al., 2000; Ma et al.,2010). Haurie suggests that oscillation mechanism in cyclical neutropenia is due to destabilization of HSCs regulatory would explain the fact that other cell lineages oscillate with the same period as cyclical neutropenia (Haurie et al., 1998; Haurie et al., 2000; Hearn et al.,1998). Hopf bifurcation phenomenon has been observed in cyclical neutropenia model which lead to periodical oscillation of neutrophil numbers and the stable periodic solution branches out from equilibrium (Haurie et al., 1998). Biological meaningfully, Lei have developed CN model by introducing granulocyte-colony stimulating factors (G-CSF) into $\mathrm{CN}$ administration with the assumption that G-CSF may decrease the amplification coefficient in neutrophil line for the treatment of cyclical neutropenia (Lei \& Mackey, 2014; Zhuge et al., 2012). Recently, the dependence of neutrophil response on the period of simulated chemotherapy and the secondary response to G-CSF adminstaration is also reported physiologically and mathematically (Haurie et al., 1998). 
Lei developed cyclical neutropenia model including G-CSF administration which can be described as follows:

$$
\begin{aligned}
& \frac{d Q}{d t}=-\left(\beta(Q)+k_{N}(N)+k_{\delta}\right) Q+2 e^{-r_{s} \tau_{s}} \beta\left(Q_{\tau_{s}}\right) Q_{\tau_{s}}, \\
& \frac{d N}{d t}=-\gamma_{N} N+A_{N} k_{N}\left(N_{\tau_{N}}\right) Q_{\tau_{N}}
\end{aligned}
$$

Wherein, HSCs exist in its proliferating phase with nonlinear rate $\beta$, and enter into $\mathrm{CN}$ compartment with nonlinear rate $k_{N}$. Commonly, both $\beta$ and $k_{N}$ are given by the Holling function which listed

$$
\begin{aligned}
& k_{N}(N)=\frac{f_{0} \theta_{1}^{m}}{\theta_{1}^{m}+N^{m}} \quad \beta(Q)=\frac{k_{c} \theta_{2}^{n}}{\theta_{2}^{n}+Q^{n}} \\
& k_{N}\left(N_{\tau_{N}}\right)=\frac{f_{0} \theta_{1}^{m}}{\theta_{1}^{m}+N\left(t-\tau_{N}\right)^{m}} \quad \beta\left(Q_{\tau_{s}}\right)=\frac{k_{c 0} \theta_{2}^{n}}{\theta_{2}^{n}+Q\left(t-\tau_{s}\right)^{n}}
\end{aligned}
$$

In model (1.1), HSCs can be self-renewal and differentiate to produce all kinds of blood cells including white blood cells, platelets and erythrocytes with rate $k_{\delta} . \gamma_{s}$ denotes apoptosis rate and $\tau_{s}$ denotes proliferation time. The delay in circulating neutrophil is $\tau_{N}$ which can be decomposed as different time period experienced, that is, the proliferation time $\tau_{N P}$ (days) of its progenitor cells and the maturation time $\tau_{N M}$ (days) of cyclical neutropenia. $\eta_{N P}$ expresses the proliferation rate and $\gamma_{0}$ is the death rate in its maturation phase. Therefore, the amplification coefficient in circulating neutropenia is

$$
A_{N}=e^{\eta_{N P} \tau_{N P}-\gamma_{0} \tau_{N M}}
$$

In this paper, we introduce state feedback control mechanism to describe system (1.1) as

$$
\begin{aligned}
& \frac{d Q}{d t}=-\left(\beta(Q)+k_{N}(N)+k_{\delta}\right) Q+2 e^{-r_{s} \tau_{s}} \beta\left(Q_{\tau_{s}}\right) Q_{\tau_{s}}+K\left(Q_{\tau_{N}}+Q_{\tau_{s}}-2 Q\right), \\
& \frac{d N}{d t}=-\gamma_{N} N+A_{N} k_{N}\left(N_{\tau_{N}}\right) Q_{\tau_{N}}
\end{aligned}
$$

Hopf bifurcation and the instability of system (1.2) may bring out the complex dynamical behavior as varying parameter continuously. However, it is tremendous rough work to work out the threshold of Hopf bifurcation as varying time delay. Recently, DDE-Biftool is developed to effectively solve the root attribution of the related characteristic equation and compute the stability of the equlibrium solution of system (1.2). Hopf bifurcation occurs as a pair of imaginary roots of the characteristic equation cross the imaginary axis from left half plane to right half plane transversally and gives rise to oscillating periodic solutions with small amplitudes. In system (1.1), Hopf bifurcation point is tracked by regarding amplification coefficient as bifurcation parameters and bistable regime of steady states and periodic solution is found by using DDE-biftool software (Engelborghs et al., 2001; Green et al., 2002).

In this paper, we explore double Hopf bifurcation point of system (1.2) by tracking the secondary Hopf bifurcation lines which form the resonant region near "death island" (Zhang \& Xu, 2013; Zhang \& Xu, 2011; Ma et al., 2009). Different features as asymptotically stability in "death island" and periodic or chaotic attractor after double Hopf bifurcation exhibit the richness of system dynamics inherently. In section 2, we discuss the stability of the related linearized system and double Hopf bifurcation point which is highlighted by "death island" is explored by using DDE-Biftool. In section 3, by using center manifold method and normal form theory, dynamics of the linearized system near the double Hopf bifurcation point is analyzed. In section 4, dynamics of nonlinear system near the double Hopf point is analyzed. Numerical simulation further verifies the classified dynamical results, and coexistence of periodical solutions and torus are detected near double Hopf bifurcation point.

\section{Double Hopf Bifurcation}

Suppose $E\left(Q^{*}, N^{*}\right)$ is a positive equilibrium solution of (1.1), by using DDE-Biftool, stability of equilibrium solution $E\left(Q^{*}, N^{*}\right)$ is computed with accuracy and Hopf bifurcation line is tracked by varying parameter continuously. We choose $\gamma_{N}=2.5, \eta_{N P}=2.542, \gamma_{0}=0.25, f_{0}=0.8, k_{\delta}=0.012, \theta_{1}=36, \theta_{2}=0.16, r_{s}=0.0631, \tau_{N P}=5, \epsilon=-0.0583, \tau_{s}=$ 2.6902 fixed and set parameter $k_{0}, \tau_{N}$ free, instability of the equilibium solution is detected after Hopf bifurcation happens. Therefore, Hopf bifurcation lines is drawn on $\left(k_{0}, \tau_{N}\right)$ plane as shown in Figure 1. It is seen that the secondary Hopf bifurcation happens at double Hopf bifurcation points which brings out the so-called "death island".

The linearized equation of system (1.2) is written as

$$
\begin{aligned}
& x^{\prime}=a_{11} x+a_{12} y+b_{1} x\left(t-\tau_{s}\right)+K\left(x\left(t-\tau_{N}\right)+x\left(t-\tau_{s}\right)-2 x\right) \\
& y^{\prime}=-\gamma_{N} y+b_{2} x\left(t-\tau_{N}\right)+b_{3} y\left(t-\tau_{N}\right)
\end{aligned}
$$


where

$$
\begin{aligned}
& a_{11}=-\beta\left(Q^{*}\right)-k_{N}\left(N^{*}\right)-k_{\delta}-\beta^{\prime}\left(Q^{*}\right) Q^{*}, \quad a_{12}=-k_{N}^{\prime}\left(N^{*}\right) Q^{*}, \\
& b_{1}=2 e^{-r_{s} \tau_{s}}\left(\beta\left(Q^{*}\right)+\beta^{\prime}\left(Q^{*}\right) Q^{*}\right), \\
& b_{3}=e^{\tau_{N P} \eta_{N P}-\gamma_{0} \tau_{N M}} k^{\prime}\left(N^{*}\right) Q^{*},
\end{aligned}
$$

The related characteristic equation of linearized system (2.1) is

$$
\Delta\left(\lambda, \gamma_{s}, \tau_{s}\right)=\left|\begin{array}{cc}
\left(a_{11}-2 K\right)-\lambda+\left(b_{1}+K\right) e^{-\lambda \tau_{s}}+K e^{-\lambda \tau_{N}} & a_{12} \\
b_{2} e^{-\lambda \tau_{N}} & -\gamma_{N}-\lambda+b_{3} e^{-\lambda \tau_{N}}
\end{array}\right|=0
$$

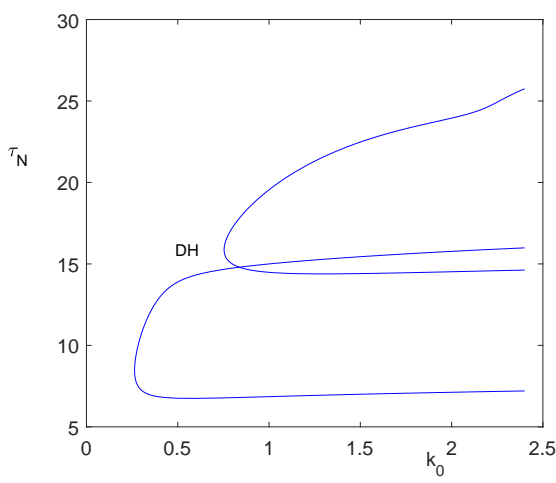

(a)

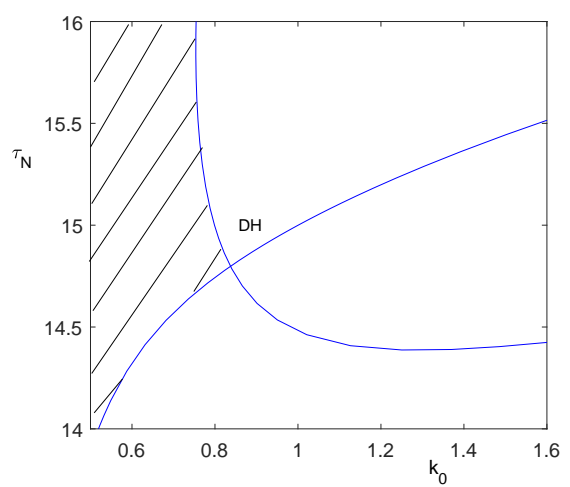

(b)

Figure 1. Double Hopf bifurcation occurs in system(1.2). (a)Hopf bifurcation lines drawn on $\left(k_{0}, \tau_{N}\right)$ parameter plane£(b) The so-called "death island" is found near double Hopf bifurcation point and denoted by the shaded regime

Roots of the characteristic equation (2.2) with zero real parts appears along Hopf bifurcation line, and one denotes $\lambda=$ $\pm i \omega_{1}$ which satisfies $\mathrm{Eq}(2.2)$ with $\omega_{1}>0$. The transversallity of Hopf bifurcation can be verified which expresses the necessary condition of stability switching of the equilibrium solution $E\left(Q^{*}, N^{*}\right)$, that is, equilibrium solution $E\left(Q^{*}, N^{*}\right)$ becomes unstable when entering into the regime enclosed and bounded by Hopf bifurcation line. The secondary Hopf bifurcation line is also detected with roots $\lambda= \pm i \omega_{2}\left(\omega_{2}>0\right)$ and the double Hopf bifurcation point is explored at critical parameters $k_{0}=0.83739666, \tau_{N}=14.79810125$. At the double Hopf point $D H$, both transversallity condition as $\left.\frac{d \mathfrak{R}(\lambda)}{d \tau_{N}}\right|_{\lambda=i \omega_{1}}<0$ and $\left.\frac{d \mathfrak{R}(\lambda)}{d \tau_{N}}\right|_{\lambda=i \omega_{2}}>0$ are satisfied. As shown in Figure 1(a), ahead of the double Hopf bifurcation point $D H$, equilibrium solution $E\left(Q^{*}, N^{*}\right)$ changes from local asymptotically stable to be unstable as increasing time delay to cross over Hopf bifurcation line, then retrieve its stability again. The stability switching of equilibrium solution $E\left(Q^{*}, N^{*}\right)$ brings out the "death island" which is shown by shaded regime in Figure 1(b) which is the amplification of Figure 1(a).

\section{Double Hopf Bifurcation}

As analyzed in section 2, Double Hopf bifurcation happened at pairs of critical values $\left(k_{c 0}, \tau_{N 0}\right)$. For convenience, set $\beta=k_{c} \beta_{1}$ hereinafter. By setting $x \rightarrow \sqrt{\epsilon} x, y \rightarrow \sqrt{\epsilon} y$ and $k_{0}=k_{c 0}+\epsilon k_{\epsilon}, \tau_{N}=\tau_{N 0}+\epsilon \tau_{\epsilon}$, system (1.2) is equivalent to

$$
\begin{aligned}
& x^{\prime}= a_{11} x+a_{12} y+b_{1} x\left(t-\tau_{s}\right)+K\left(x\left(t-\tau_{N 0}\right)+x\left(t-\tau_{s}\right)+2 x\right)+\epsilon l_{11} x+\epsilon l_{12} x\left(t-\tau_{s}\right) \\
&+K\left(x\left(t-\tau_{N}\right)-x\left(t-\tau_{N 0}\right)\right)+f\left(x, x\left(t-\tau_{s}\right), y\right), \\
& y^{\prime}=\left.-\gamma_{N} y+b_{2} x\left(t-\tau_{N 0}\right)+b_{3} y\left(t-\tau_{N 0}\right)+b_{2}\left(x\left(t-\tau_{N}\right)\right)-x\left(t-\tau_{N 0}\right)\right) \\
&+b_{3}\left(y\left(t-\tau_{N}\right)-y\left(t-\tau_{N 0}\right)\right)+\epsilon l_{21} x\left(t-\tau_{N 0}\right) \\
&+\epsilon l_{22} y\left(t-\tau_{N 0}\right)+g\left(x\left(t-\tau_{N}\right), y, y\left(t-\tau_{N}\right)\right),
\end{aligned}
$$

where

$$
\begin{array}{ll}
a_{11}=-k_{c 0} \beta_{1}\left(Q^{*}\right)-k_{N}\left(N^{*}\right)-k_{\delta}-k_{c 0} \beta_{1}^{\prime}\left(Q^{*}\right) Q^{*}, & a_{12}=-k_{N}^{\prime}\left(N^{*}\right) Q^{*}, \\
b_{1}=2 e^{-r_{s} \tau_{s}}\left(k_{c 0} \beta_{1}\left(Q^{*}\right)+k_{c 0} \beta^{\prime}\left(Q^{*}\right) Q^{*}\right), & b_{2}=e^{\tau_{N P} \eta_{N P}-\gamma_{0} \tau_{N M}} k\left(N^{*}\right), \\
b_{3}=e^{\tau_{N P} \eta_{N P}-\gamma_{0} \tau_{N M}} k^{\prime}\left(N^{*}\right) Q^{*}, & l_{11}=-k_{\epsilon} \beta_{1}\left(Q^{*}\right)-k_{\epsilon} \beta_{1}^{\prime}\left(Q^{*}\right) Q^{*}, \\
l_{12}=2 e^{-r_{s} \tau_{s}}\left(k_{\epsilon} \beta_{1}\left(Q^{*}\right)+k_{\epsilon} \beta^{\prime}\left(Q^{*}\right) Q^{*}\right), & l_{21}=\gamma_{0} \tau_{\epsilon} e^{\tau_{N P} \eta_{N P}-\gamma_{0} \tau_{N M}} k_{N}\left(N^{*}\right), \\
l_{22}=\gamma_{0} \tau_{\epsilon} e^{\tau_{N P} \eta_{N P}-\gamma_{0} \tau_{N M}} k_{N}^{\prime}\left(N^{*}\right) Q^{*} &
\end{array}
$$


and

$$
\begin{aligned}
& f(x, x(t-\left.\left.\tau_{s}\right), y\right)=2 \sqrt{\epsilon} k_{c 0} e^{-r_{s} \tau_{s}} \beta_{1}^{\prime}\left(Q^{*}\right) x^{2}\left(t-\tau_{s}\right)+\sqrt{\epsilon} k_{c 0} e^{-r_{s} \tau_{s}} \beta_{1}^{\prime \prime}\left(Q^{*}\right) Q^{*} x^{2}\left(t-\tau_{s}\right) \\
&+2 \epsilon \sqrt{\epsilon} k_{\epsilon} e^{-r_{s} \tau_{s}} \beta_{1}^{\prime}\left(Q^{*}\right) x^{2}\left(t-\tau_{s}\right)-\frac{1}{2} \sqrt{\epsilon} k_{c 0} \beta_{1}^{\prime \prime}\left(Q^{*}\right) Q^{*} x^{2}-\epsilon \frac{1}{6} k_{c 0} \beta_{1}^{\prime \prime \prime}\left(Q^{*}\right) Q^{*} x^{3} \\
&-\sqrt{\epsilon} k_{c 0} \beta_{1}^{\prime}\left(Q^{*}\right) x^{2}-\frac{1}{2} \epsilon k_{c 0} \beta_{1}^{\prime \prime}\left(Q^{*}\right) x^{3}-\frac{1}{2} \epsilon \sqrt{\epsilon} k_{\epsilon} \beta_{1}^{\prime \prime}\left(Q^{*}\right) Q^{*} x^{2}-\epsilon \sqrt{\epsilon} k_{\epsilon} \beta_{1}^{\prime}\left(Q^{*}\right) x^{2} \\
&-\sqrt{\epsilon} k_{N}^{\prime}\left(N^{*}\right) x y-\frac{1}{2} \epsilon k_{N}^{\prime \prime}\left(N^{*}\right) x y^{2}-\frac{1}{2} \sqrt{\epsilon} k_{N}^{\prime \prime}\left(N^{*}\right) Q^{*} y^{2}-\frac{1}{6} \epsilon k_{N}^{\prime \prime \prime}\left(N^{*}\right) Q^{*} y^{2} \\
&+\epsilon \sqrt{\epsilon} k_{\epsilon} e^{-r_{s} \tau_{s}} \beta_{1}^{\prime \prime}\left(Q^{*}\right) Q^{*} x^{2}\left(t-\tau_{s}\right)+\epsilon k_{c 0} e^{-r_{s} \tau_{s}} \beta_{1}^{\prime \prime}\left(Q^{*}\right) x^{3}\left(t-\tau_{s}\right) \\
&+\epsilon \frac{1}{3} k_{c 0} e^{-r_{s} \tau_{s}} \beta_{1}^{\prime \prime \prime}\left(Q^{*}\right) Q^{*} x^{3}\left(t-\tau_{s}\right) \\
& g\left(x\left(t-\tau_{N}\right), y, y\left(t-\tau_{N}\right)\right)=\sqrt{\epsilon} e^{\tau_{N P} \eta_{N P}-\gamma_{0} \tau_{N M}} k_{N}^{\prime}\left(N^{*}\right) x\left(t-\tau_{N}\right) y\left(t-\tau_{N}\right) \\
&+\frac{1}{2} \epsilon e^{\tau_{N P} \eta_{N P}-\gamma_{0} \tau_{N M}} k_{N}^{\prime \prime}\left(N^{*}\right) x\left(t-\tau_{N}\right) y^{2}\left(t-\tau_{N}\right) \\
&-\epsilon \sqrt{\epsilon} e^{\tau_{N N} \eta_{N P}-\gamma_{0} \tau_{N M}} k_{N}^{\prime}\left(N^{*}\right) x\left(t-\tau_{N}\right) y\left(t-\tau_{N}\right) \\
&+\frac{1}{2} \sqrt{\epsilon} e^{\tau_{N P} \eta_{N P}-\gamma_{0} \tau_{N M}} k_{N}^{\prime \prime}\left(N^{*}\right) Q^{*} y^{2}\left(t-\tau_{N}\right) \\
&+\frac{1}{6} \epsilon e^{\tau_{N P} \eta_{N P}-\gamma_{0} \tau_{N M}} k_{N}^{\prime \prime \prime}\left(N^{*}\right) Q^{*} y^{3}\left(t-\tau_{N}\right)
\end{aligned}
$$

Choose phase space as $C=C\left(\left[-\tau_{N}, 0\right], R^{2}\right)$, which is a Banach space composed as all continuous and differential function by mapping from $\left[-\tau_{N}, 0\right] \rightarrow R^{2}$. For every $\phi \in C$, one can define the linear operator as

$$
L(0) \phi=\int_{-\tau_{N}}^{0}[d \eta(\theta)] \phi(\theta),
$$

where $\eta:\left[-\tau_{N}, 0\right] \rightarrow R^{2} \times R^{2}$ is a bounded variation function in $\left[-\tau_{N}, 0\right]$ and

$$
d \eta(\theta)=\left[\begin{array}{cc}
\left(a_{11}-2 K\right) \delta(\theta)+\left(b_{1}+K\right) \delta\left(\theta+\tau_{s}\right)+K \delta\left(\theta+\tau_{N 0}\right) & a_{12} \delta(\theta) \\
b_{2} \delta\left(\theta+\tau_{N 0}\right) & -\gamma_{N} \delta(\theta)+b_{3} \delta\left(\theta+\tau_{N 0}\right)
\end{array}\right] d \theta
$$

Furthermore, we define

$$
L\left(k_{\epsilon}, \tau_{\epsilon}\right)=\int_{-\tau_{N}}^{0}\left[d \eta_{1}\left(\theta, k_{\epsilon}\right)+d \eta_{2}\left(\theta, \tau_{\epsilon}\right)\right] \phi(\theta),
$$

with

$$
d \eta_{1}\left(\theta, k_{\epsilon}\right)=\left[\begin{array}{cc}
l_{11} \delta(\theta)+l_{12} \delta\left(\theta+\tau_{s}\right) & 0 \\
0 & 0
\end{array}\right]
$$

and

$$
d \eta_{2}\left(\theta, \tau_{\epsilon}\right)=\left[\begin{array}{cc}
K \delta\left(\theta+\tau_{N}\right)-K \delta\left(\theta+\tau_{N 0}\right) & 0 \\
b_{2} \delta\left(\theta+\tau_{N}\right)-\left(b_{2}+\epsilon l_{21}\right) \delta\left(\theta+\tau_{N 0}\right) & b_{3} \delta\left(\theta+\tau_{N}\right)-\left(b_{3}+\epsilon l_{22}\right) \delta\left(\theta+\tau_{N 0}\right)
\end{array}\right]
$$

Suppose the linear operator at double Hopf bifurcation point $\left(k_{c 0}, \tau_{N 0}\right)$ is

$$
H(0) \phi= \begin{cases}\frac{d \phi}{d \theta}, & \theta \in\left[-\tau_{N}, 0\right) \\ L(0) \phi, & \theta=0\end{cases}
$$

and

$$
H\left(k_{\epsilon}, \tau_{\epsilon}\right)=\left\{\begin{array}{cc}
0, & \theta \in\left[-\tau_{N}, 0\right) \\
L\left(k_{\epsilon}, \tau_{\epsilon}\right) \phi, & \theta=0
\end{array}\right.
$$

The nonlinear operator $R$ is defined as

$$
R(\phi)= \begin{cases}0, & \theta \in\left[-\tau_{N}, 0\right) \\ F(\phi), & \theta=0\end{cases}
$$

and $F(\phi)=\left(\begin{array}{c}f\left(\phi_{1}(0), \phi_{1}\left(\tau_{s}\right), \phi_{2}(0)\right) \\ g\left(\phi_{1}\left(-\tau_{N}\right), \phi_{2}(0), \phi_{2}\left(-\tau_{N}\right)\right)\end{array}\right)$.

System (3.1) can be rewritten as its operator form as (Hale, 2003)

$$
u^{\prime}(t)=H(0) u_{t}+H\left(k_{\epsilon}, \tau_{\epsilon}\right) u_{t}+R u_{t}
$$

with $u=(x, y)^{T}$, and $u_{t}=u(t+\theta)$, for $-\tau_{N} \leq \theta<0$. For $\Psi \in C^{*}=C\left(\left[0, \tau_{N}\right], R^{2}\right)$ define the adjoint operator $H^{*}(0)$ of operator $H(0)$ £and adjoint operator $H^{*}\left(k_{\epsilon}, \tau_{\epsilon}\right)$ of $H\left(k_{\epsilon}, \tau_{\epsilon}\right)$, respectively as

$$
H^{*}(0) \psi= \begin{cases}-\frac{d \psi}{d s}, & 0<s \leq \tau_{N}, \\ \int_{-\tau_{N}}^{0} d \eta^{T}(s) \psi(-s), & s=0\end{cases}
$$


and

$$
H^{*}\left(k_{\epsilon}, \tau_{\epsilon}\right) \psi= \begin{cases}-\frac{d \psi}{d s}, & 0<s \leq \tau_{N}, \\ \int_{-\tau_{N}}^{0}\left[d \eta_{1}^{T}\left(s, k_{\epsilon}\right)+d \eta_{2}^{T}\left(s, \tau_{\epsilon}\right)\right] \psi(-s), & s=0,\end{cases}
$$

For $\phi \in C, \psi \in C^{*}$, define the belinear form (, ) : $C^{*} \times C \rightarrow R$ as

$$
<\psi, \phi>=\bar{\psi}^{T}(0) \phi(0)+\int_{-\tau_{s}}^{0} \bar{\psi}^{T}\left(\xi+\tau_{s}\right) R_{1} \phi(\xi) d \xi+\int_{-\tau_{N}}^{0} \bar{\psi}^{T}\left(\xi+\tau_{N}\right) R_{2} \phi(\xi) d \xi
$$

By the above discussion, there are two pairs of imaginary roots at the double Hopf bifurcation point $\left(k_{\epsilon}, \tau_{\epsilon}\right)$, and other characteristic roots with negative real parts. We denote the collection set $\Lambda=\left\{i \omega_{1},-i \omega_{1}, i \omega_{2},-i \omega_{2}\right\}$ and decompose the phase space $C$ to be the direct sum of its subspaces as $C=P_{\Lambda} \oplus Q_{\Lambda}$, with $P_{\Lambda}$ being the characteristic space of $\Lambda$ and $Q_{\Lambda}$ being the corresponding complement subspace. System (3.1) is an ordinary differential system on the Banach space $C$ of functions from $\left[-\tau_{N}, 0\right]$ to $R^{2}$, which is bounded and continuous on $\left(-\tau_{N}, 0\right)$ with a possible jump discontinuity at 0 (Buono et al., 2003). We choose

$$
\begin{aligned}
& \Phi(\theta)=\left(q_{1}(\theta), \bar{q}_{1}(\theta), q_{2}(\theta), \bar{q}_{2}(\theta)\right), \\
& \Psi(\theta)=\left(q_{1}^{*}(\theta), \bar{q}_{1}^{*}(\theta), q_{2}^{*}(\theta), \bar{q}_{2}^{*}(\theta)\right),
\end{aligned}
$$

as the bases of $P_{\Lambda}$ and $P_{\Lambda}^{*}$, where

$$
q_{1}(\theta)=\left(\begin{array}{c}
\gamma_{N}+i \omega_{1}-b_{3} e^{-i \omega_{1} \tau_{N 0}} \\
b_{2} e^{-i \omega_{1} \tau_{N 0}} \\
\gamma_{N}+i \omega_{2}-b_{3} e^{-i \omega_{2} \tau_{N 0}} \\
b_{2} e^{-i \omega_{2} \tau_{N 0}}
\end{array}\right) e^{i \omega_{1} \theta},
$$

for $-\tau_{N} \leq \theta<0$, are respectively the eigenvectors of operator $H(0)$ with respect to the characteristic root $i \omega_{1}, i \omega_{2}$, and the eigenvectors corresponding to adjoint operator $A^{*}(0)$ with respect to $-i \omega_{1}$ and $-i \omega_{2}$ are respectively as

$$
q_{1}^{*}(s)=l_{1}\left(\begin{array}{c}
-b_{2} e^{i \omega_{1} \tau_{N}} \\
q_{11}^{*}(s)=l_{2}\left(\begin{array}{c}
\omega_{1}+\left(b_{1}+K\right) e^{i \omega_{1} \tau_{s}}+K e^{i \omega_{1} \tau_{N}} \\
-b_{2} e^{i \omega_{2} \tau_{N}} \\
a_{11}+i \omega_{2}+\left(b_{1}+K\right) e^{i \omega_{2} \tau_{s}}+K e^{i \omega_{2} \tau_{N}}
\end{array}\right) e^{i \omega_{1} s}, \\
\end{array}\right.
$$

for $0 \leq s \leq \tau_{N}$, where

$$
l_{1}=\frac{e^{i \omega_{0} \tau_{N 0}}}{s_{1} \tau_{N 0}+s_{2} \tau_{s}+s_{3}}, \quad l_{2}=\frac{1}{v_{1} \tau_{N 0}+v_{2} \tau_{s}+v_{3}}
$$

with

$$
\begin{aligned}
s_{1}= & -K \omega_{1}^{2}+\left(-(2 i) K \gamma_{N}+(2 i) K b_{3} e^{i \omega_{1} \tau_{N 0}}-i a_{12} b_{2}\right) \omega_{1}+K \gamma_{N}^{2} \\
& +a_{12} b_{2} K b_{3} e^{i \omega_{1} \tau_{N 0}} \gamma_{N}+K b_{3}^{2} e^{2 i \omega_{1} \tau_{N 0}}, \\
s_{2}= & \left(i b_{2} K e^{i \omega_{1} \tau_{s}}+i b_{2} b_{1} e^{i \omega_{1} \tau_{s}}\right) \omega_{1}+\left(-b_{2} K e^{i \omega_{1} \tau_{s}}-b_{2} b_{1} e^{i \omega_{1} \tau_{s}}\right) \gamma_{N} \\
& +b_{2} K b_{3} e^{i \omega_{1}\left(\tau_{N 0}+\tau_{s}\right)}+b_{2} b_{1} b_{3} e^{i \omega_{1}\left(\tau_{N 0}+\tau_{s}\right)}, \\
s_{3}= & -e^{-i \omega_{1} \tau_{N 0}} \omega_{1}^{2}+\left((-2 i) e^{-i \omega_{1} \tau_{N 0}} \gamma_{N}+2 i b_{3}\right) \omega_{1} \\
& +e^{-i \omega_{1} \tau_{N 0}} \gamma_{N}^{2}-2 \gamma_{N} b_{3}+b_{3}^{2} e^{i \omega_{1} \tau_{N 0}}+a_{12} b_{2}, \\
v_{1}= & -K \omega_{2}^{2}+\left(-(2 i) K \gamma_{N}+(2 i) K b_{3} e^{i \omega_{2} \tau_{N 0}}-i a_{12} b_{2}\right) \omega_{2}+K \gamma_{N}^{2} \\
& +a_{12} b_{2} K b_{3} e^{i \omega_{2} \tau_{N 0}} \gamma_{N}+K b_{3}^{2} e^{2 i \omega_{2} \tau_{N 0}}, \\
v_{2}= & \left(i b_{2} K e^{i \omega_{2} \tau_{s}}+i b_{2} b_{1} e^{i \omega_{2} \tau_{s}}\right) \omega_{2}+\left(-b_{2} K e^{i \omega_{2} \tau_{s}}-b_{2} b_{1} e^{i \omega_{2} \tau_{s}}\right) \gamma_{N} \\
& +b_{2} K b_{3} e^{i \omega_{2}\left(\tau_{N 0}+\tau_{s}\right)}+b_{2} b_{1} b_{3} e^{i \omega_{2}\left(\tau_{N 0}+\tau_{s}\right)}, \\
v_{3}= & -e^{-i \omega_{2} \tau_{N 0}} \omega_{2}^{2}+\left((-2 i) e^{-i \omega_{2} \tau_{N 0}} \gamma_{N}+2 i b_{3}\right) \omega_{2} \\
& +e^{-i \omega_{2} \tau_{N 0}} \gamma_{N}^{2}-2 \gamma_{N} b_{3}+b_{3}^{2} e^{i \omega_{2} \tau_{N 0}}+a_{12} b_{2},
\end{aligned}
$$

It can be verified that

$$
<q_{1}^{*}, q_{1}>=<q_{2}^{*}, q_{2}>=1
$$

is satisfied and further we can prove that

$$
\begin{aligned}
& <q_{1}^{*}, \bar{q}_{1}>=<q_{1}^{*}, q_{2}>=<q_{1}^{*}, \bar{q}_{2}>=0, \\
& <q_{2}^{*}, \bar{q}_{2}>=<q_{2}^{*}, q_{1}>=<q_{2}^{*}, \bar{q}_{1}>=0 .
\end{aligned}
$$

It is noticed that

$$
\begin{array}{lll}
A q_{1}(\theta)=i \omega_{1} q_{1}(\theta), & A q_{2}(\theta)=i \omega_{2} q_{2}(\theta), & -\tau_{N} \leq \theta \leq 0, \\
A^{*} q_{1}^{*}(s)=-i \omega_{1} q_{1}^{*}(s), & A^{*} q_{2}^{*}(s)=-i \omega_{2} q_{2}^{*}(s), \quad 0 \leq s \leq \tau_{N} .
\end{array}
$$


Let $z=\left(z_{1}, \bar{z}_{1}, z_{2}, \bar{z}_{2}\right)^{T}$, for every $u_{t} \in C$, and $v_{t} \in Q_{\Lambda} \cap C^{\prime}\left(\left[-\tau_{N}, 0\right], R^{2}\right)$, we have the expression $u_{t}=\Phi z+v_{t}$ and the linearized system corresponds to (3.11) is

$$
\dot{u}_{t}=H(0) u_{t}+H\left(k_{\epsilon}, \tau_{\epsilon}\right) u_{t},
$$

with the reduction form on its center manifold as

$$
\begin{aligned}
z^{\prime} & =B z+<\bar{\Psi}, H\left(k_{\epsilon}, \tau_{\epsilon}\right) u_{t}> \\
& =B z+\bar{\Psi}^{T}(0) A\left(k_{\epsilon}, \tau_{\epsilon}\right)\left(\Phi z+v_{t}\right),
\end{aligned}
$$

where

$$
B=\left(\begin{array}{cccc}
i \omega_{1} & 0 & 0 & 0 \\
0 & -i \omega_{1} & 0 & 0 \\
0 & 0 & i \omega_{2} & 0 \\
0 & 0 & 0 & -i \omega_{2}
\end{array}\right)
$$

The matrix $B$ generates the torus group $T^{2}=S^{1} \times S^{1}$ whose action on $C^{2}$ is given by

$$
\left(\theta_{1}, \theta_{2}\right)\left(z_{1}, z_{2}\right)=\left(e^{i \theta_{1}} z_{1}, e^{i \theta_{2}} z_{2}\right)
$$

Then the $T^{2}$-equivalent normal form, which is truncated to the quadratic order, becomes

$$
\left(\begin{array}{l}
\dot{z}_{1} \\
\dot{z}_{2}
\end{array}\right)=\left(\begin{array}{cc}
i \omega_{1} & 0 \\
0 & i \omega_{2}
\end{array}\right)\left(\begin{array}{l}
z_{1} \\
z_{2}
\end{array}\right)+\left(\begin{array}{c}
\epsilon k_{\epsilon} c_{1}^{1} z_{1}+\epsilon \tau_{\epsilon} c_{2}^{1} z_{1} \\
\epsilon k_{\epsilon} c_{1}^{2} z_{1}+\epsilon \tau_{\epsilon} c_{2}^{2} z_{1}
\end{array}\right)
$$

with

$$
\begin{aligned}
& c_{1}^{1}=\left(\left(-(2 i) \omega_{1}-2 \gamma_{N}\right) \exp \left(\left(-r_{s}-i \omega_{1}\right) \tau_{s}-i \omega_{1} \tau_{N 0}\right)+2 \exp \left(\left(-r_{s}-i \omega_{1}\right) \tau_{s}-(2 i) \omega_{1} \tau_{N 0}\right) b_{3}\right. \\
& \left.+\left(\gamma_{N}+i \omega_{1}\right) \exp \left(-i \omega_{1} \tau_{N 0}\right)-\exp \left(-(2 i) \omega_{1} \tau_{N 0}\right) b_{3}\right) l_{1} \epsilon b_{2}\left(\beta_{1}^{\prime}\left(Q^{*}\right) Q^{*}+\beta_{1}\left(Q^{*}\right)\right) \text {, } \\
& c_{2}^{1}=-\epsilon l_{1}\left(\gamma_{0} A_{N} b_{3}\left(Q^{*} b_{2} k_{N}\left(N^{*}\right)-b_{3} k_{N}\left(N^{*}\right)\right)\left(b_{1}+K\right) \exp \left(-i \omega_{1}\left(2 \tau_{N 0}+\tau_{s}\right)\right)\right. \\
& +\left(k_{N} A_{N} \gamma_{0}\left(\gamma_{N}+i \omega_{1}\right) b_{3}+\omega_{1}\left(i \gamma_{N}-\omega_{1}\right) b_{2}\right)\left(b_{1}+K\right) \exp \left(-i \omega_{1}\left(\tau_{N 0}+\tau_{s}\right)\right) \\
& +\left(-k_{N}\left(N^{*}\right) A_{N}\left(-\omega_{1}^{2}+\left(i \gamma_{N}-i a_{11}\right) \omega_{1}-a_{11} \gamma_{N}\right) \gamma_{0} b_{3}\right. \\
& \left.+\omega_{1}\left(i \omega_{1}^{2}+\omega_{1}\left(\gamma_{N}-a_{11}\right)+i a_{11} \gamma_{N}\right) b_{2}\right) \exp \left(-i \omega_{1} \tau_{N 0}\right)+\left(A _ { N } \left(k_{N}\left(N^{*}\right)\left(i \omega_{1}-a_{11}\right) b_{3}\right.\right. \\
& \left.+\left(-i k_{N}^{\prime}\left(N^{*}\right) Q^{*} b_{2}+i K k_{N}\left(N^{*}\right)\right) \omega_{1}+k_{N}^{\prime}\left(N^{*}\right) Q^{*} b_{2} a_{11}+K \gamma_{N} k_{N}\left(N^{*}\right)\right) \gamma_{0} \exp \left(-(2 i) \omega_{1} \tau_{N 0}\right) \\
& \left.\left.+\operatorname{Kexp}\left(-(3 i) \omega_{1} \tau_{N 0}\right)\left(-\gamma_{0} A_{N} b_{3} k_{N}\left(N^{*}\right)+\left(k_{N}^{\prime}\left(N^{*}\right) \gamma_{0} Q^{*} A_{N}+i \omega_{1}\right) b_{2}\right)\right) b_{3}\right), \\
& c_{1}^{2}=\left(\left(-(2 i) \omega_{1}-2 \gamma_{N}\right) \exp \left(\left(-r s-i \omega_{1}\right) \tau_{s}-i \omega_{1} \tau_{N 0}\right)+2 \exp \left(\left(-r_{s}-i \omega_{1}\right) \tau_{s}-(2 i) \omega_{1} \tau_{N 0}\right) b_{3}\right. \\
& \left.+\left(\gamma_{N}+i \omega_{1}\right) \exp \left(-i \omega_{1} \tau_{N 0}\right)-\exp \left(-(2 i) \omega_{1} \tau_{N 0}\right) b_{3}\right) l_{1} \epsilon b_{2}\left(\beta_{1}^{\prime}\left(Q^{*}\right) Q^{*}+\beta_{1}\right), \\
& c_{2}^{2}=-l_{2} \epsilon\left(k_{N}\left(N^{*}\right) A_{N} b_{3}\left(b_{1}+K\right) \gamma_{0}\left(\gamma_{N}+i \omega_{1}\right) \exp \left(-i \omega_{1} \tau_{N 0}-i \omega_{2} \tau_{s}\right)\right. \\
& +\gamma_{0} A_{N} b_{3}\left(Q^{*} b_{2} k_{N}^{\prime}\left(N^{*}\right)-b_{3} k_{N}\left(N^{*}\right)\right)\left(b_{1}+K\right) \exp \left(-(2 i) \omega_{1} \tau_{N 0}-i \omega_{2} \tau_{s}\right) \\
& +K \gamma_{0} A_{N} b_{3}\left(Q^{*} b_{2} k_{N}^{\prime}\left(N^{*}\right)-b_{3} k_{N}\left(N^{*}\right)\right) \exp \left(-i \tau_{N 0}\left(2 \omega_{1}+\omega_{2}\right)\right) \\
& +\omega_{2}\left(i \gamma_{N}-\omega_{2}\right)\left(b_{1}+K\right) b_{2} \exp \left(-i \omega_{2}\left(\tau_{N 0}+\tau_{s}\right)\right) \\
& +k_{N}\left(N^{*}\right) A_{N} K b_{3} \gamma_{0}\left(\gamma_{N}+i \omega_{1}\right) \exp \left(-i \tau_{N 0}\left(\omega_{1}+\omega_{2}\right)\right) \\
& -A_{N} b_{3}\left(i \omega_{2}-a_{11}\right)\left(Q^{*} b_{2} k_{N}^{\prime}\left(N^{*}\right)-b_{3} k_{N}\left(N^{*}\right)\right) \gamma_{0} \exp \left(-(2 i) \omega_{1} \tau_{N 0}\right) \\
& \left.-k_{N}\left(N^{*}\right) A_{N}\left(\left(i \gamma_{N}-\omega_{1}\right) \omega_{2}-a_{11} \gamma_{N}+i \omega_{1}\right)\right) b_{3} \gamma_{0} \exp \left(-i \omega_{1} \tau_{N 0}\right) \\
& \left.+\omega_{2}\left(\left(i \omega_{2}^{2}+\omega_{2}\left(\gamma_{N}-a_{11}\right)+i a_{11} \gamma_{N}\right) \exp \left(-i \omega_{2} \tau_{N 0}\right)+i \operatorname{Kexp}\left(-(3 i) \omega_{2} \tau_{N 0}\right) b_{3}\right) b_{2}\right),
\end{aligned}
$$

In polar coordinate $\left(\rho_{1}, \theta_{1}, \rho_{2}, \theta_{2}\right)$, we express the associated amplitude equation generated from system (3.19) as

$$
\begin{aligned}
& \rho_{1}^{\prime}=\epsilon \mathfrak{R}\left(k_{\epsilon} c_{1}^{1}+\tau_{\epsilon} c_{2}^{1}\right) \rho_{1}, \\
& \rho_{2}^{\prime}=\epsilon \mathfrak{R}\left(k_{\epsilon} c_{1}^{2}+\tau_{\epsilon} c_{2}^{2}\right) \rho_{2}
\end{aligned}
$$




\section{Center Manifold Reduction}

Furthermore, consider the nonlinear system

$$
\dot{u}_{t}=H(0) u_{t}+R u_{t}
$$

It is directly computed that the reduction form derived from Eq.(4.1) is

$$
\left\{\begin{aligned}
z_{1}^{\prime} & =i \omega_{1} z+\bar{q}_{1}^{* T}(0) F\left(\Phi z+v_{t}\right) \\
& =i \omega_{1} z+\bar{q}_{1}^{* T}(0) \hat{f}\left(z_{1}, \bar{z}_{1}, z_{2}, \bar{z}_{2}\right) \\
z_{2}^{\prime} & =i \omega_{2} z+\bar{q}_{2}^{* T}(0) F\left(\Phi z+v_{t}\right) \\
& =i \omega_{2} z+\bar{q}_{2}^{* T}(0) \hat{f}\left(z_{1}, \bar{z}_{1}, z_{2}, \bar{z}_{2}\right) \\
v_{t} & =A v_{t}+(I-\Pi) X_{0}\left(F\left(\Phi z+v_{t}\right)\right)
\end{aligned}\right.
$$

with $I$ identity and $\Pi$ is the projection mapping from $C\left([-\tau, 0], R^{2}\right)$ to the subspace $P_{\Lambda}$, and $X_{0}$ denotes

$$
X_{0}=\left\{\begin{array}{l}
0, \theta \in\left[-\tau_{N}, 0\right) \\
I, \theta=0
\end{array}\right.
$$

where

$$
\hat{f}\left(z_{1}, \bar{z}_{1}, z_{2}, \bar{z}_{2}, v_{t}\right)=F\left(2 \Re\left(z_{1} q_{1}(\theta)\right)+2 \Re\left(z_{2} q_{2}(\theta)\right)+v_{t}(\theta)\right)
$$

We write $v_{t}$ into its Taylor series

$$
v_{t}=w\left(z_{1}, \bar{z}_{1}, z_{2}, \bar{z}_{2}\right)=\sum_{i+j+k+l \geq 2} w_{i j k l} z_{1}^{i} \bar{z}_{1}^{j} z_{2}^{k} \bar{z}_{2}^{l}
$$

with coefficient vector $w_{i j k l}$ only depend on $\theta$. We now expand $\hat{f}$ into the Taylor series and

$$
\begin{aligned}
& g_{1}\left(z_{1}, \bar{z}_{1}, z_{2}, \bar{z}_{2}\right)=\bar{q}_{1}^{* T}(0) \hat{f}\left(z_{1}, \bar{z}_{1}, z_{2}, \bar{z}_{2}, v_{t}\right)=\sum_{i+j+k+l \geq 2}^{3} g_{i j k l}^{1} z_{1}^{i} \bar{z}_{1}^{j} z_{2}^{k} \bar{z}_{2}^{l}, \\
& g_{2}\left(z_{1}, \bar{z}_{1}, z_{2}, \bar{z}_{2}\right)=\bar{q}_{2}^{* T}(0) \hat{f}\left(z_{1}, \bar{z}_{1}, z_{2}, \bar{z}_{2}, v_{t}\right)=\sum_{i+j+k+l \geq 2}^{3} g_{i j k l}^{2} z_{1}^{i} z_{1}^{j} z_{2}^{k} \bar{z}_{2}^{l}
\end{aligned}
$$

From system (4.1), we obtain

$$
v_{t}^{\prime}=A v_{t}+\left\{\begin{array}{cr}
-2 \mathfrak{R}\left\{p_{1}^{T}(0) \hat{f}\left(z_{1}, \bar{z}_{1}, z_{2}, \bar{z}_{2}\right) q_{1}(\theta)\right\} & -2 \mathfrak{R}\left\{p_{2}^{T}(0) \hat{f}\left(z_{1}, \bar{z}_{1}, z_{2}, \bar{z}_{2}\right) q_{2}(\theta)\right\}, \\
-2 \mathfrak{R}\left\{p_{1}^{T}(0) \hat{f}\left(z_{1}, \bar{z}_{1}, z_{2}, \bar{z}_{2}\right) q_{1}(\theta)\right\} & -2 \mathfrak{R}\left\{p_{2}^{T}(0) \hat{f}\left(z_{1}, \bar{z}_{1}, z_{2}, \bar{z}_{2}\right) q_{2}(\theta)\right\} \\
+\hat{f}\left(z_{1}, \bar{z}_{1}, z_{2}, \bar{z}_{2}\right), & \theta=0
\end{array}\right.
$$

The first equation in Eq.(4.6) can be rewritten as

$$
w^{\prime}\left(z_{1}, \bar{z}_{1}, z_{2}, \bar{z}_{2}\right)=A w\left(z_{1}, \bar{z}_{1}, z_{2}, \bar{z}_{2}\right)+B\left(z_{1}, \bar{z}_{1}, z_{2}, \bar{z}_{2}\right),-\tau_{N} \leq \theta<0
$$

where

$$
\begin{aligned}
& B\left(z_{1}, \bar{z}_{1}, z_{2}, \bar{z}_{2}\right)=-2 \mathfrak{R}\left\{p_{1}^{T}(0) \hat{f}\left(z_{1}, \bar{z}_{1}, z_{2}, \bar{z}_{2}\right) q_{1}(\theta)\right\}-2 \mathfrak{R}\left\{p_{2}^{T}(0) \hat{f}\left(z_{1}, \bar{z}_{1}, z_{2}, \bar{z}_{2}\right) q_{2}(\theta)\right\} \\
= & \sum_{i+j+k+l \geq 2} B_{i j k k} z_{1}^{i} \bar{z}_{1}^{j} z_{2}^{k} \bar{z}_{2}^{l},
\end{aligned}
$$

with the coefficients $B_{i j k l}=\left(B_{i j k l}^{1}(\theta), B_{i j k l}^{2}(\theta)\right)^{T}$.

It can be derived from Eq.(4.6) that

$$
\begin{aligned}
\left(A-2 i \omega_{1}\right) w_{2000} & =-B_{2000}(\theta), \\
A w_{1100} & =-B_{1100}(\theta), \\
\left(A+2 i \omega_{1}\right) w_{0200}= & -B_{0200}(\theta), \\
\left(A-i \omega_{1}-i \omega_{2}\right) w_{1010}= & -B_{1010}(\theta), \\
\left(A-i \omega_{1}+i \omega_{2}\right) w_{1001}= & -B_{1001}(\theta), \\
\left(A+i \omega_{1}-i \omega_{2}\right) w_{0110}= & -B_{0110}(\theta), \\
\left(A+i \omega_{1}+i \omega_{2}\right) w_{0101}= & -B_{0101}(\theta), \\
\left(A-2 i \omega_{2}\right) w_{0020}= & -B_{0020}(\theta), \\
A w_{0011}= & -B_{0011}(\theta), \\
\left(A+2 i \omega_{2}\right) w_{0002} & =-B_{0002}(\theta),
\end{aligned}
$$


Taking account of formula of operator $A$, we have

$$
\begin{aligned}
& \frac{d w_{2000}}{d \theta}=2 i \omega_{1} w_{2000}(\theta)-B_{2000}(\theta), \\
& \frac{d w_{1100}}{d \theta}=-B_{1100}(\theta), \\
& \frac{d w_{0200}}{d \theta}=-2 i \omega_{1} w_{0200}(\theta)-B_{0200}(\theta), \\
& \frac{d w_{1010}}{d \theta}=i\left(\omega_{1}+\omega_{2}\right) w_{1010}(\theta)-B_{1010}(\theta), \\
& \frac{d w_{1001}}{d \theta}=i\left(\omega_{1}-\omega_{2}\right) w_{1001}(\theta)-B_{1001}(\theta), \\
& \frac{d w_{0110}}{d \theta}=i\left(-\omega_{1}+\omega_{2}\right) w_{0110}(\theta)-B_{0110}(\theta), \\
& \frac{d w_{0101}}{d \theta}=-i\left(\omega_{1}+\omega_{2}\right) w_{0101}(\theta)-B_{0101}(\theta), \\
& \frac{d w_{0020}}{d \theta}=2 i \omega_{2} w_{0020}(\theta)-B_{0020}(\theta), \\
& \frac{d w_{0011}}{d \theta}=-B_{0011}(\theta), \\
& \frac{d w_{0002}}{d \theta}=-2 i \omega_{2} w_{0002}(\theta)-B_{0002}(\theta),
\end{aligned}
$$

Integrating Eq.(4.9) under the initial condition given be the second equation in Eq.(4.6), we can compute the coefficient vectors $w_{i j k l}(\theta)$ with $i+j+k+l=2$.

Using the standard technique as invertible parameter-dependent complex coordinates transformation which is introduced in (Buono et al., 2003), the normal form at $\left(k_{c 0}, \tau_{N 0}\right)$ of Eq.(4.1) is described as

$$
\begin{aligned}
& z_{1}^{\prime}=i \omega_{1} z_{1}+B_{11} z_{1}^{2} \bar{z}_{1}+B_{12} z_{1} z_{2} \bar{z}_{2}, \\
& z_{2}^{\prime}=i \omega_{2} z_{2}+B_{21} z_{1} \bar{z}_{1} z_{2}+B_{22} z_{2}^{2} \bar{z}_{2}
\end{aligned}
$$

In polar coordination $\left(\rho_{1}, \theta_{1}, \rho_{2}, \theta_{2}\right)$, the amplitude equation generated from Eq.(4.10) is

$$
\left\{\begin{array}{l}
\rho_{1}^{\prime}=\rho_{1}\left(\mathfrak{R}\left(B_{11}\right) \rho_{1}^{2}+\mathfrak{R}\left(B_{12}\right) \rho_{2}^{2}\right), \\
\rho_{2}^{\prime}=\rho_{2}\left(\mathfrak{R}\left(B_{21}\right) \rho_{1}^{2}+\mathfrak{R}\left(B_{22}\right) \rho_{2}^{2}\right),
\end{array}\right.
$$

Combining the results of Eqs(3.19) and Eqs(4.10), the formal normal form arising from system (4.11) is

$$
\begin{aligned}
& z_{1}^{\prime}=i \omega_{1} z_{1}+\epsilon c_{1}^{1} k_{\epsilon} z_{1}+\epsilon c_{1}^{2} \tau_{\epsilon} z_{1}+B_{11} z_{1}^{2} \bar{z}_{1}+B_{12} z_{1} z_{2} \bar{z}_{2}, \\
& z_{2}^{\prime}=i \omega_{2} z_{2}+\epsilon c_{2}^{1} k_{\epsilon} z_{2}+\epsilon c_{2}^{2} \tau_{\epsilon} z_{2}+B_{21} z_{1} \bar{z}_{1} z_{2}+B_{22} z_{2}^{2} \bar{z}_{2},
\end{aligned}
$$

Using polar coordinate transformation $z_{1}=\rho_{1} e^{i \theta_{1}}, z_{2}=\rho_{2} e^{i \theta_{2}}$, system (4.12) is transformed into

$$
\left\{\begin{array}{l}
\rho_{1}^{\prime}=\rho_{1}\left(\mu_{1}+\mathfrak{R}\left(B_{11}\right) \rho_{1}^{2}+\mathfrak{R}\left(B_{12}\right) \rho_{2}^{2}\right), \\
\rho_{2}^{\prime}=\rho_{2}\left(\mu_{2}+\mathfrak{R}\left(B_{21}\right) \rho_{1}^{2}+\mathfrak{R}\left(B_{22}\right) \rho_{2}^{2}\right), \\
\theta_{1}^{\prime}=\omega_{1}+v_{1}+\mathfrak{J}\left(B_{11}\right) \rho_{1}^{2}+\mathfrak{J}\left(B_{12}\right) \rho_{2}^{2}, \\
\theta_{2}^{\prime}=\omega_{2}+v_{2}+\mathfrak{J}\left(B_{21}\right) \rho_{1}^{2}+\mathfrak{J}\left(B_{22}\right) \rho_{2}^{2},
\end{array}\right.
$$

with $\mu_{i}=\epsilon\left(\mathfrak{R}\left(c_{i}^{1}\right) k_{\epsilon}+\mathfrak{R}\left(c_{i}^{2}\right) \tau_{\epsilon}\right), v_{i}=\epsilon\left(\mathfrak{J}\left(c_{i}^{1}\right) k_{\epsilon}+\mathfrak{J}\left(c_{i}^{2}\right) \tau_{\epsilon}\right)$ for $i=1,2$. Using Maple software, we compute the coefficients $g_{i j l k}$ with $i+j+k+l=2,3$, and further obtain the following formulas (Zhang, S. \& Xu, J., 2011).

$$
\begin{aligned}
& B_{11}= g_{2100}^{1}+\frac{i}{\omega_{1}} g_{1100}^{1} g_{2000}^{1}+\frac{i}{\omega_{2}}\left(g_{1010}^{1} g_{1100}^{2}-g_{1001}^{1} \bar{g}_{1100}^{2}\right)-\frac{i}{2 \omega_{1}+\omega_{2}} g_{0101}^{1} \bar{g}_{0200}^{2} \\
&-\frac{i}{2 \omega_{1}-\omega_{2}} g_{0110}^{1} g_{2000}^{2}-\frac{i}{\omega_{1}}\left|g_{1100}^{1}\right|^{2}-\frac{2 i}{3 \omega_{1}}\left|g_{0200}^{1}\right|^{2}, \\
& B_{12}= g_{1011}^{1}+\frac{i}{\omega_{2}}\left(g_{1010}^{1} g_{0011}^{2}-g_{1001}^{1} \bar{g}_{0011}^{2}\right)+\frac{i}{\omega_{1}}\left(2 g_{2000}^{1} g_{0011}^{1}-g_{1100}^{1} \bar{g}_{0011}^{1}-g_{0011}^{1} \bar{g}_{0110}^{2}\right. \\
&\left.\quad-g_{0011}^{1} g_{1010}^{2}\right)-\frac{2 i}{\omega_{1}+2 \omega_{2}} g_{0002}^{1} \bar{g}_{0101}^{2}-\frac{2 i}{\omega_{1}-2 \omega_{2}} g_{0020}^{1} g_{1001}^{2}-\frac{i}{2 \omega_{1}-\omega_{2}}\left|g_{0110}^{1}\right|^{2}-\frac{i}{2 \omega_{1}+\omega_{2}}\left|g_{0101}^{1}\right|^{2}, \\
& B_{21}= g_{1110}^{2}+\frac{i}{\omega_{1}}\left(g_{1100}^{1} g_{1010}^{2}-\bar{g}_{1100}^{1} g_{0110}^{2}\right)+\frac{i}{\omega_{2}}\left(2 g_{0020}^{2} g_{1100}^{2}-g_{0011}^{2} \bar{g}_{1100}^{2}-g_{1010}^{1} g_{1100}^{2}\right. \\
&\left.-\bar{g}_{1001}^{2} g_{1100}^{2}\right)+\frac{2 i}{2 \omega_{1}-\omega_{2}} g_{0110}^{1} g_{2000}^{2}-\frac{2 i}{2 \omega_{1}+\omega_{2}}\left(\bar{g}_{0101}^{1} g_{0200}^{2}-\frac{i}{2 \omega_{2}-\omega_{1}}\left|g_{1001}^{2}\right|^{2}-\frac{i}{\omega_{1}+2 \omega_{2}}\left|g_{0101}^{2}\right|^{2},\right. \\
& B_{22}=g_{0021}^{2}+\frac{i}{\omega_{1}}\left(g_{0011}^{1} g_{1010}^{2}-\bar{g}_{0011}^{1} g_{0110}^{2}\right)+\frac{i}{\omega_{2}} g_{0011}^{2} g_{0020}^{2}-\frac{i}{2 \omega_{2}-\omega_{1}} g_{0020}^{1} g_{1001}^{2} \\
&-\frac{i}{2 \omega_{2}+\omega_{1}} \bar{g}_{0002}^{1} g_{0101}^{2}-\frac{i}{\omega_{2}}\left|g_{0011}^{2}\right|^{2}-\frac{2 i}{3 \omega_{2}}\left|g_{0002}^{2}\right|^{2}
\end{aligned}
$$

At double Hopf point DH with $Q^{*}=0.3082205820115, N^{*}=388.4842602444987, \omega_{1}=0.254524564411299, \omega_{2}=$ 0.633751246398309 , we compute the coefficients $g_{i j l k}^{1,2},(i+j+k+l=2,3)$. Therefore, by formula(4.14), choose $\epsilon=0.1$, 
then we have

$$
\begin{aligned}
B_{11}= & -0.6051712720 e-1+0.7735627 e-4 i+0.5601811760 e-2 k_{\epsilon} \\
& +(0.3431392376 e-1 i) k_{\epsilon}+(0.1393876593 e-2 i) k_{\epsilon}^{2} \\
& +0.1581514771 e-1 k_{\epsilon}^{2}, \\
B_{12}= & -0.5174126005 e-1+.2503938024 i+0.4929740694 e-1 k_{\epsilon} \\
& +(0.3335168000 e-1 i) k_{\epsilon}+0.1430425584 e-2 k_{\epsilon}^{2} \\
& +(0.1482060556 e-2 i) k_{\epsilon}^{2}, \\
B_{21}= & 0.3923165406 e-1+.1303176163 i+0.2227270962 e-1 k_{\epsilon} \\
& -(0.1269551832 e-1 i) k_{\epsilon}-(0.4852214180 e-2 i) k_{\epsilon}^{2} \\
& +0.1030896439 e-2 k_{\epsilon}^{2}, \\
B_{22}= & 0.4434841977 e-1+0.8857002742 e-1 i+0.1000013764 e-1 k_{\epsilon} \\
& -(0.5651717652 e-2 i) k_{\epsilon}-(0.2466158270 e-3 i) k_{\epsilon}^{2} \\
& +0.2884712704 e-3 k_{\epsilon}^{2}
\end{aligned}
$$

We also have

$$
\begin{aligned}
\mu_{1}= & -0.1240211011 e-2 k_{\epsilon}+(0.1962466074 e-2 i) k_{\epsilon} \\
& -(0.1702332949 e-2 i) \tau_{\epsilon}+0.2827534980 e-2 \tau_{\epsilon} \\
& -(0.7571216173 e-4 i) \tau_{\epsilon}^{2}-0.354986150 e-5 \tau_{\epsilon}^{2}, \\
\mu_{2}= & 0.8371586097 e-3 k_{\epsilon}+(0.1476100446 e-3 i) k_{\epsilon} \\
& -(0.6445732784 e-2 i) \tau_{\epsilon}+0.2543586790 e-2 \tau_{\epsilon} \\
& -(0.6272308346 e-4 i) \tau_{\epsilon}^{2}-0.6170908018 e-4 \tau_{\epsilon}^{2}
\end{aligned}
$$

By time continuous transformation with direction preserved, system (4.13) is equivalent to

$$
\begin{aligned}
& \rho_{1}^{\prime}=\rho_{1}\left(a+\frac{\mathfrak{R}\left(B_{11}\right)}{\left|B_{11}\right|} \rho_{1}^{2}+\frac{\mathfrak{R}\left(B_{12}\right)}{\left|B_{11}\right|} \rho_{2}^{2}\right), \\
& \rho_{2}^{\prime}=\rho_{2}\left(b+\frac{\mathfrak{R}\left(B_{21}\right)}{\left|B_{11}\right|} \rho_{1}^{2}+\frac{\mathfrak{R}\left(B_{22}\right)}{\left|B_{11}\right|} \rho_{2}^{2}\right) .
\end{aligned}
$$

with $a=\frac{\mathfrak{R}\left(\mu_{1}\right)}{\left|B_{11}\right|}, b=\frac{\mathfrak{R}\left(\mu_{2}\right)}{\left|B_{11}\right|}$.

System (4.17) may have equilibrium solutions $\left(\rho_{1}^{*}, 0\right),\left(0, \rho_{2}^{*}\right)$ and $\left(\rho_{1}^{*}, \rho_{2}^{*}\right)$ and stability of equilibrium solution can be derived via computation of its corresponding Jacobin determinant. Therefore, based on system(4.16), we deduce the following results

(I)Neutral saddle bifurcation of equilibrium $\left(\rho_{1}^{*}, 0\right)$ happens if $\mu_{2}+\left(2 B_{11}+B_{21}\right) \rho_{1}^{* 2}=0$;

(II)Neutral saddle bifurcation of equilibrium solution $\left(0, \rho_{2}^{*}\right)$ happens if $\mu_{1}+\left(B_{12}+2 B_{22}\right) \rho_{2}^{* 2}=0$;

(III) The equlibrium solution $\left(\rho_{1}^{*}, \rho_{2}^{*}\right)$ loses its stability through line $H L 3: B_{11} \rho_{1}^{* 2}+B_{22} \rho_{2}^{* 2}=0$ and correspondingly, the associated torus of system (4.17) getting into torus instability state.

As shown in Figure2, system (4.17) has the equilibrium solution $\left(0, \rho_{2}^{*}\right)$ with parameter pairs $\left(k_{c}, \tau_{N}\right)$ chosen in the regime above boundary line $L 1$ in green color, and the regime bounded by line in red color and line in black color exhibits torus solution. We discuss system dynamics in detail with the partition of plane into different regimes. The stable periodic solution observed in regime $I, V I$ is shown in Figure3(a)-(f). The periodic solution exhibited in regime $I$ further bifurcates into quasi-periodical solution after crossing neutral saddle line $H L 1$ shown in cyan color. The scenarios of the bifurcating quasi-periodical solutions observed in regimes from $(I I)$ to $V$ is plotted as shown in Figure4. Due to neutral line $H L 2$ in purple color, system may have multi attractors coexistence in regime $I V$. We also obtain the observed coexisted attractors in regime $I I$ and $V$ alike, as shown in Figure5.

\section{Discussion}

A blood cell model which composed of two compartment cells known as hematopoietic stem cells and its relationship with neutrophil was investigated. The dysfunction in regulatory control process of blood cell production induce the observed hematological disease and cyclical neutropenia model including G-CSF adminstration was developed. As system state dependent delay reflects different time stage during feedback regulation process, we introduced state feedback control with time delay originally arising in system which is subtle disturbance around stationary equilibrium solution. We use DDE-Biftool software to explore double Hopf bifurcation of system via tracking second Hopf bifurcaton lines as varying parameters continuously and dynamics near double Hopf point was discussed. A classified method of dynamics near double Hopf point was developed by using center manifold theory. The analytical norm form near double Hopf point is computed based on fundamental theory of functional differential equation and the observed numerical simulation results were in coincidence with dynamics of the classified method which further verified the correctness of theoretical analytical results. 


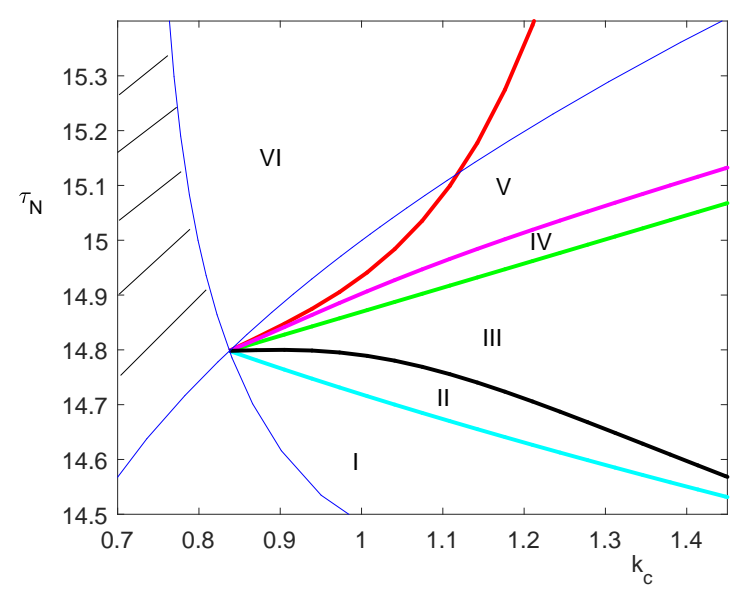

Figure 2. The dynamical classify results near the double Hopf point $D H$ in parameter space $\left(k_{0}, \tau_{N}\right)$ plane

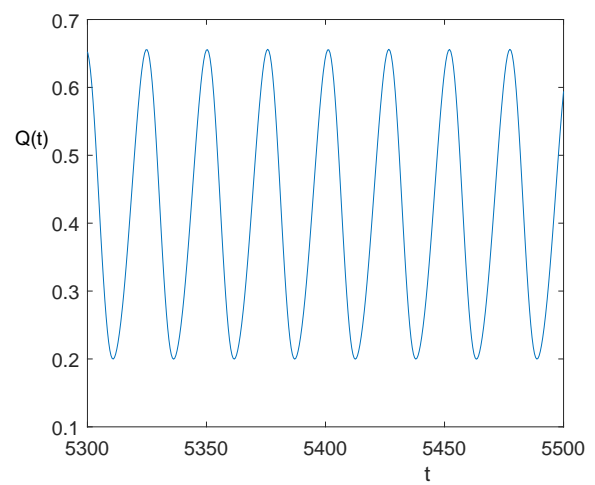

(a)

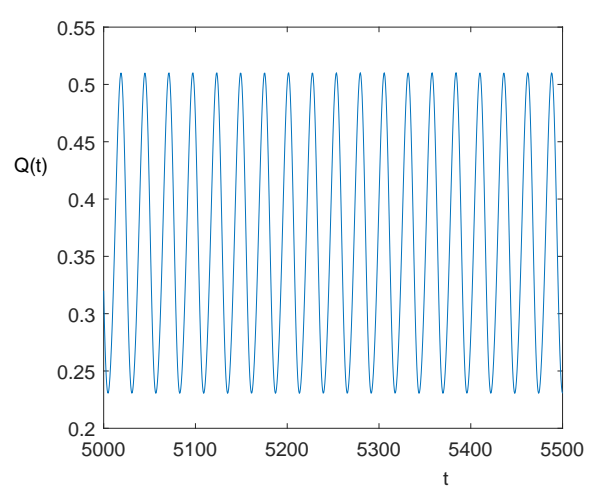

(c)

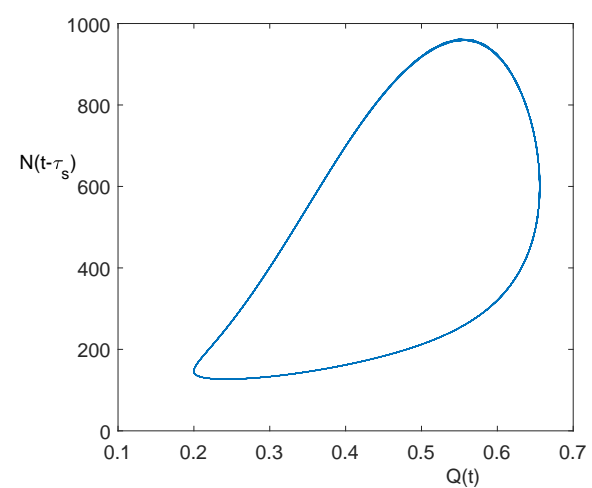

(b)

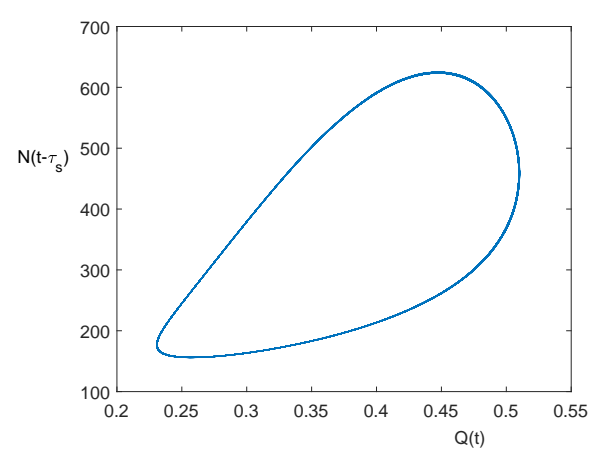

(d)

Figure 3. Periodical solution of system (1.2).(a) Phase portraits of solution with parameter $k_{c}=1.1, \tau_{N}=14.6$; (b) Time series solution with parameter $k_{c}=1.1, \tau_{N}=14.6$; (c) Phase portraits of solution with parameter $k_{c}=0.9, \tau_{N}=15.1$; (d) Time series solution with parameter $k_{c}=0.9, \tau_{N}=15.1$ 


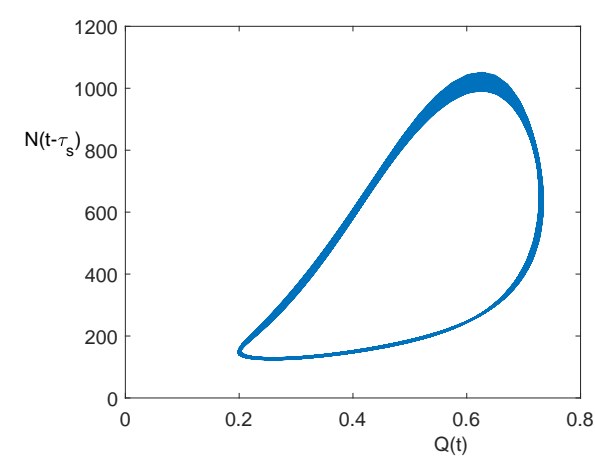

(a)

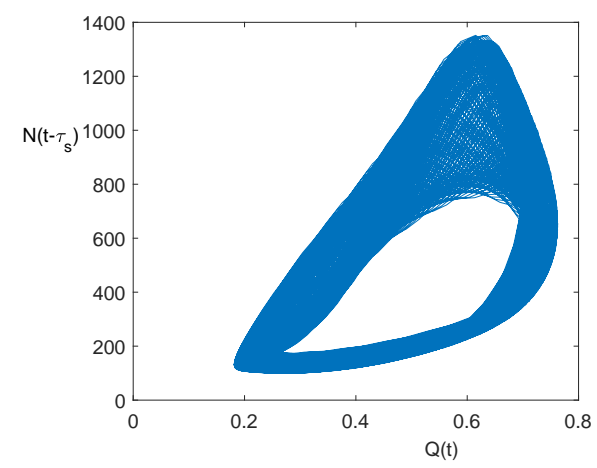

(c)

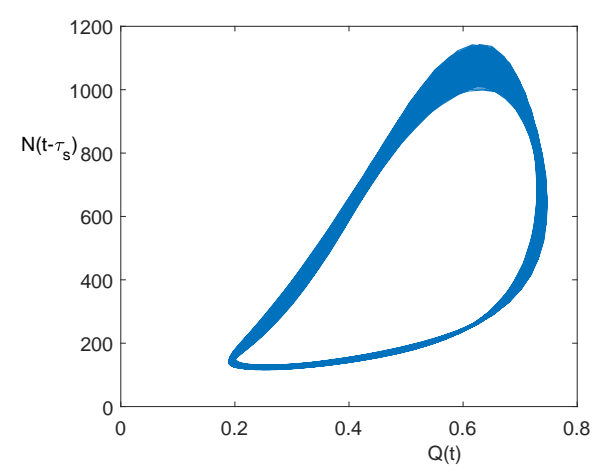

(b)

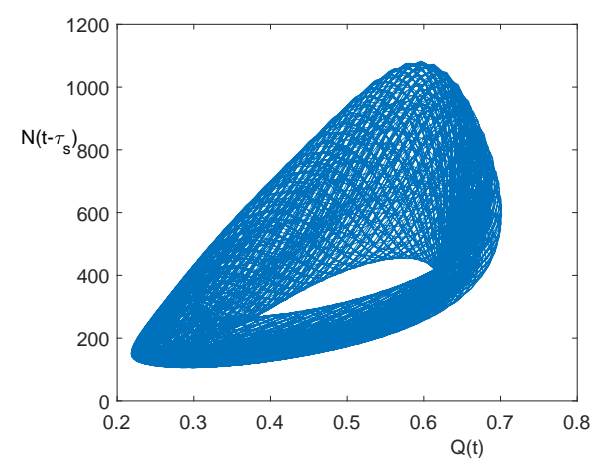

(d)

Figure 4. The numerical simulation results of system (1.2) near the double Hopf point $D H$. (a)Torus at parameter pairs $\left(k_{c}, \tau_{N}\right)$ lying in regime $I I$; (b) Torus at parameter pairs $\left(k_{c}, \tau_{N}\right)$ lying in regime $I I I$; (c)Torus at parameter pairs $\left(k_{c}, \tau_{N}\right)$ lying in regime $I V ;(\mathrm{d})$ Torus at parameter pairs $\left(k_{c}, \tau_{N}\right)$ lying in regime $V$

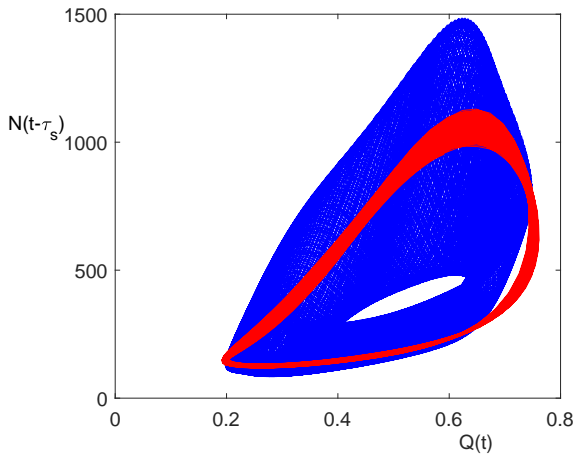

(a)

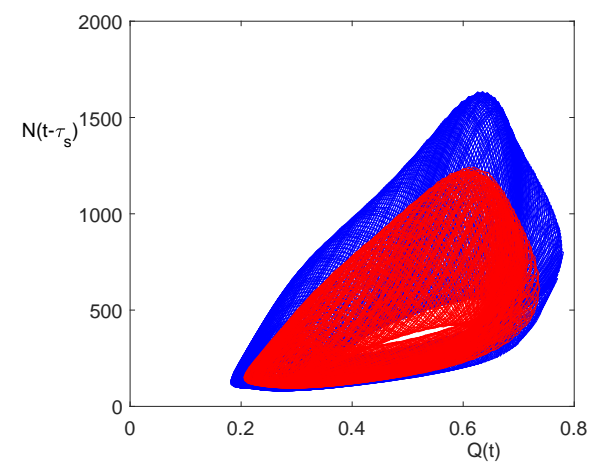

(b)

Figure 5. The coexistence phenomena of torus solutions. (a)Chosen parameter pairs $\left(k_{c}, \tau_{N}\right)$ lying in regime $I I I$; (b) Chosen parameter pairs $\left(k_{c}, \tau_{N}\right)$ lying regime $V$ 


\section{References}

Bernard, S., Bélair, J., \& Mackey, M. C. (2003). Oscillation in cyclical neutropenia: new evidence based on mathematical modelling. Journal of Theory Biology, 223, 293-298.

Buono, P. L., \& Bélair, J. (2003). Restrictions and unfolding of double Hopf bifurcation in functional differentail equations. J. Differentail Equations, 189, 234-266.

Engelborghs, K., Luzyanina, T., \& Samacy, G. (2001). DDE-BIFTOOL v2.00: a Matlab package for bifurcation analysis of delay differential equations. Technical Report TW-330, Department of Compute Science, K.U. Leuven, Belgium. http://www.cs.kuleuven.ac.be/ koen/delay/ddfbiftool.shtml.

Fowler, A. C., \& Mackey, M. C. (2002). Relaxation oscillations in a class of delay differential equations. SIAM J. Appl. Math, 63(1), 299-323.

Green, K., Krauskopf, B., \& Engelborghs, K. (2002). Bistability and torus break-up in a semiconductor lase with phaseconjugate feedback. Phys.D, 173, 114-129.

Hale, J. (2003). Theory of Functional Differential Equations, World Publishing Corporation, Beijing, China.

Fowler, A. C. (1997). Mathematical Models in the Applied Sciences. Cambridge Texts in Applied Mathematics, Cambridge University Press, Cambridge.

Haurie, C., Dale, D. C., \& Mackey, M. C. (1998). Cyclical neutropenia and other periodic hematological diseases: a review of mechanisms and mathematical models. Blood, 92, 2629-2640.

Haurie, C., Dale, D. C., Rudnicki, R., \& Mackey, M. C. (2000). Modelling complex neutrophil dynamics in the grey collie. Journal of Thoery Biology 24, 505-519.

Hearn, T., Haurie, C., \& Mackey, M. C.(1998). Cyclical neutropenia and the peripherial control of white blood cell production. Journal of Theory Biology, 192, 167-181.

Lei, J. Z., \& Mackey, M. C. (2014). Understanding and treating cytopenia through mathematical modelling, 844, 279302, Springer New York.

Mackey, M. C. (1978). Unified hypothesis for the origin of aplastic anemia and periodic hematopoiesis. Blood 51(5), 941C956.

Ma, S. Q., Wang, X. H., Lei, J. H., \& Feng, Z. S. (2010). Dynamics of the delay hematological cell model. International Journal of Biomathematics, 3, 105-125.

Ma, S. Q., Feng, Z. S., \& Lu, Q. S. (2009). Dynamics and double Hopf bifurtions of the Rose-Hindmarsh model with time delay. International Journal of Bifurcation and Chaos, 19, 1-19.

Pujo-Menjouet, L., \& Mackey, M. C. (2004). Contribution to the study of periodic chromic myelogenous leukemia. Comptes Rendus Biol., 327, 235-244.

Pujo-Menjouet, L., Bernard, S., \& Mackey, M. C. (2005). Long period oscillation in a $G_{0}$ model of hematopoietic stem cells. SIAM J. Appl. Dyn.Syst., 4(2), 312-332.

Santillan, M., Mahaffy, J. M., Bélair, J., \& Mackey, M. C. (2000). Regulation of platelet production: The normal response to perturbation and cyclical platelet disease. Journal of Theory Biology, 206, 585-603.

Zhang, S., \& Xu, J. (2013). Quasiperiodic motion induced by heterogeneous delays in a simplified internet congestion control model. Nonlineat Analysis: Real World Applications, 14, 661-670.

Zhang, S., \& Xu, J. (2011). Time-varying delayed feedback control for an internet congestion control model. Discrete and Continuous Dynamical Systems Series B., 2011, 653-665.

Zhuge, C. J., Lei, J. Z., \& Mackey, M. C. (2012). Neutrophil dynamics in response to chemotherapy and G-SCF. Journal of Theory Biology, 293(1), 111-120.

\section{Copyrights}

Copyright for this article is retained by the author(s), with first publication rights granted to the journal.

This is an open-access article distributed under the terms and conditions of the Creative Commons Attribution license (http://creativecommons.org/licenses/by/4.0/). 